\title{
A NEW APPROACH OF CONCURRENT CALL HANDLING PROCEDURE IN MOBILE NETWORKS
}

\author{
P. K. Guha Thakurta ${ }^{1}$ Misha hungyo ${ }^{2}$ Jahnavi Katikitala $^{3}$ and \\ Darakshan Anwar ${ }^{4}$ \\ 1,2,3,4 Department of Computer Science and Engineering, \\ National Institute of Technology, Durgapur, WestBengal \\ ${ }^{1}$ parag.nitdgp@gmail.com, \\ ${ }^{2}$ mkongharegmail.com, \\ 3 jahnavi6392@gmail.com, \\ ${ }^{4}$ dara.singh09876@gmail.com
}

\begin{abstract}
A new approach for handling concurrent call requests by a number of senders in mobile cellular network is proposed in this paper. The concurrent access is resolved with the introduction of semaphore concept. The several factors are identified to establish a priority factor $(P F)$ for the sender node. Based on this PF value, the right sender is selected by the receiver in case of concurrent requests. This selection algorithm executes in linear time. The effectiveness of the proposed model is analyzed with the introduction of progress graph.
\end{abstract}

\section{KEYWORDS}

Concurrent call requests, semaphore, mobile networks, progress graph

\section{INTRODUCTION}

In mobile cellular networks, mobile nodes communicate with each other using multi-hop links. This structure is stationary because there are base stations (nodes) in every cell. Each node in the network has call forwarding capability to other nodes [5]. Till date, various routing strategies have been designed to address the problem of finding routing path with efficient congestion control technique. Simultaneously, it needs a more efficient methodology to increase throughput and reduce network latency at the same time. To provide the efficient routing strategy, the nodes are grouped into manageable clusters based on different parameters and the Quality of Service $(Q o S)$ availability of each node. Here, the cluster heads $(\mathrm{CHs})$ play the role of local coordinators and they maintain the $Q o S$ values of all cluster members. Using this information, a $C H$ can forward the calls to the corresponding destinations [4]. Thus, the network design problem associated with this is to find a least cost or a maximum revenue network, reducing the redundant admitted calls.

Once the clusters are formed and maintained, these can be used to handle incoming call requests. When a mobile node sends a call request, the call is forwarded through the path as: $C S \rightarrow B S S \rightarrow$ Leader $S \rightarrow$ Leader $R \rightarrow B S R \rightarrow C R$ [1], where $C S, B S S$ and LeaderS denote the sender's node, its corresponding $B S$ and the $C H$ of that cluster respectively. Similar notations are used for

David C. Wyld et al. (Eds) : COSIT, DMIN, SIGL, CYBI, NMCT, AIAPP - 2014 
the receiver part, i.e., $C R, B S R$ and LeaderR respectively. The concurrent call handling procedure for multiple numbers of sender nodes under such scenario is not discussed. Moreover, the number of unsuccessful attempts by a sender node is not taken into consideration in this procedure and so, no priority is assigned on this basis. This may lead to indefinite waiting time for such node.

In this paper, a new approach for handling concurrent call requests by the multiple numbers of sender nodes in mobile cellular networks is proposed. The concurrent access is resolved with the introduction of semaphore [2] concept. The priority factor $(P F)$ is calculated by the receiver in case of multiple numbers of senders. Here, PF is dependent on the available bandwidth $(B W)$ to handle call request, timestamp $\left(t_{s}\right)$, and repeated request factor $(R R F)$ of the sender node. With the help of this $R R F$ value, the number of unsuccessful attempts by a sender node is defined. Thus the node with the highest $P F$ is selected as the right one. In order to avoid indefinite waiting time for the selection by receiver node, ts and $R R F$ values of the specific sender whose requests had been processed already, are set to zero. With the introduction of $P F$, the proposed model in this work executes in linear time. In addition, the effectiveness of the proposed model is analyzed using progress graph.

The remainder of the paper is organized as follows. In section II, the proposed model is described. Next, the advantage of this work is concluded in section III.

\section{PROPOSED MODEL}

The model proposed in this work obeys the following system model.

\subsection{System Model and assumptions}

Suppose, there are three clusters of nodes shown in Fig. 1. Two of them represent the sender's clusters among three, whereas the remaining one denotes the receiver's cluster. The sender nodes and are trying concurrently to connect with the receiver through their respective $\mathrm{CHs}$.

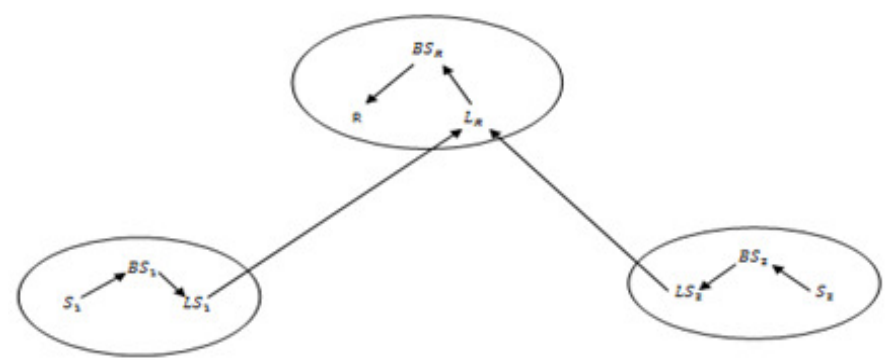

Fig. 1: Concurrent call requests from multiple sender's to a receiver

Now, the receiver node has a responsibility to select one of them having the highest priority factor (PF). This PF is dependent on three following factors.

(a) Bandwidth $(B W)$ : It is the amount of available $B W$ to handle the call request. Generally, it depends on the underlying hardware architecture and the network operating system used by the sender node.

(b) Timestamp: The $t_{s}$ of any call request is determined by the call submission time in the system and it is measured as the time recorded by the system clock. The request with least timestamp would become as the oldest call. 
(c) Repeated Request Factor $(R R F)$ : It is defined as the number of unsuccessful connection establishment by the sender node. Initially, it is set to 0 and is incremented by 1 after each unsuccessful attempt. Whenever the receiver selects the specific sender node for the connection establishment, then $R R F$ is again set as 0 for that sender. The node having greater $R R F$ value is considered as higher priority node. As available bandwidth limits the number of call requests that can be processed, so $P F$ is directly proportional to bandwidth $B W$. With the help of $R R F$ factor, a preference is given to a sender node which is calling repeatedly. So, a node with greater $R R F$ value has a greater $P F$ value. Also, preference must be given to an older request. With the help of timestamp, we select the oldest request. So, $P F$ is inversely proportional to timestamp. Thus the terminology $P F$ is defined as the ratio of the product of $B W$ and $R R F$ to $t_{\boldsymbol{s}^{*}}$ It is expressed as $P F=(B W \times R R F) / t_{s}$.

\subsection{Proposed Functional Model}

The model proposed in this paper considers the situation described in Fig. 1. The sender $S_{i}$ sets its semaphores to busy state just before sending the requests. Then it checks the receiver's status whether it is busy or idle. If the receiver is busy, the leader of $S_{i}$ records $t_{s_{i}}$ and corresponding $\mathrm{RRF}_{i}$ of the sender node and subsequently store them into a generic linked list.

The linked lists are represented with following fields - (a) for the leader of the receiver node: sender's id, the PF value and address to the next node, (b) for the leader of the sender node: sender's id, RRF of $S_{i}, t_{s}$ value and address to the next node. The linked representations are shown in Fig. 2 for Fig. 1.

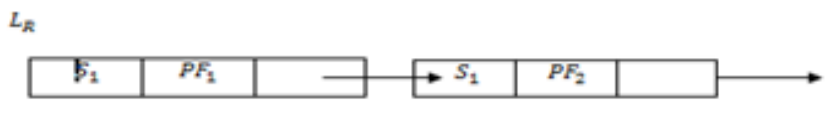

(a)

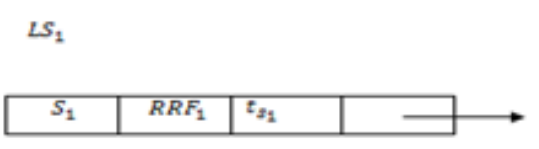

(b)

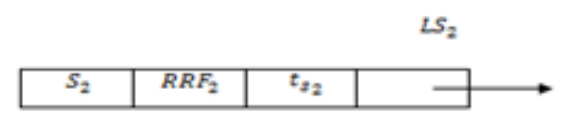

(s)

Fig. 2: Initial Linked Representation: (a) Linked List for $L_{R}$

(b) Linked List for $L S_{1}$ (c) Linked List for $L S_{2}$

the semaphore of $R$ indicates idle, the $L_{R}$ calculates the PF of the senders $S_{i}$ that has been sending call requests and stores them into its own linked list. The receiver node then selects the sender that has maximum PF from the linked list and grants its requests. After the call has been granted, the $t_{s_{i}}$ and the $\mathrm{RRF}_{i}$ of $S_{i}$ are set again to initial values. This goes on dynamically for every node and the procedure is described by the following algorithm.

\subsection{Algorithm:}

Input: ${ }^{B W_{i}}$, initial values: $t_{s_{i}}=0, \mathrm{RRF}_{i}=0$, flag $=$ FALSE. Output: $\max \left[P F\left(S_{i}\right)\right]$ 
Declaration: R=Receiver; $S_{i}=i^{\text {th }}$ Sender ; Avail $_{i}=$ Semaphore of $S_{i} ;$ Avail $_{R}$ i =Semaphore of R; $T S_{\text {tot }}=$ total timestamp; $T S_{n \boldsymbol{}}=$ new Timestamp; ${ }^{T S_{\text {pre }}=\text { previous }}$

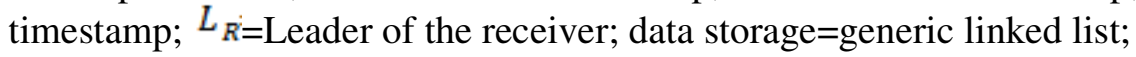

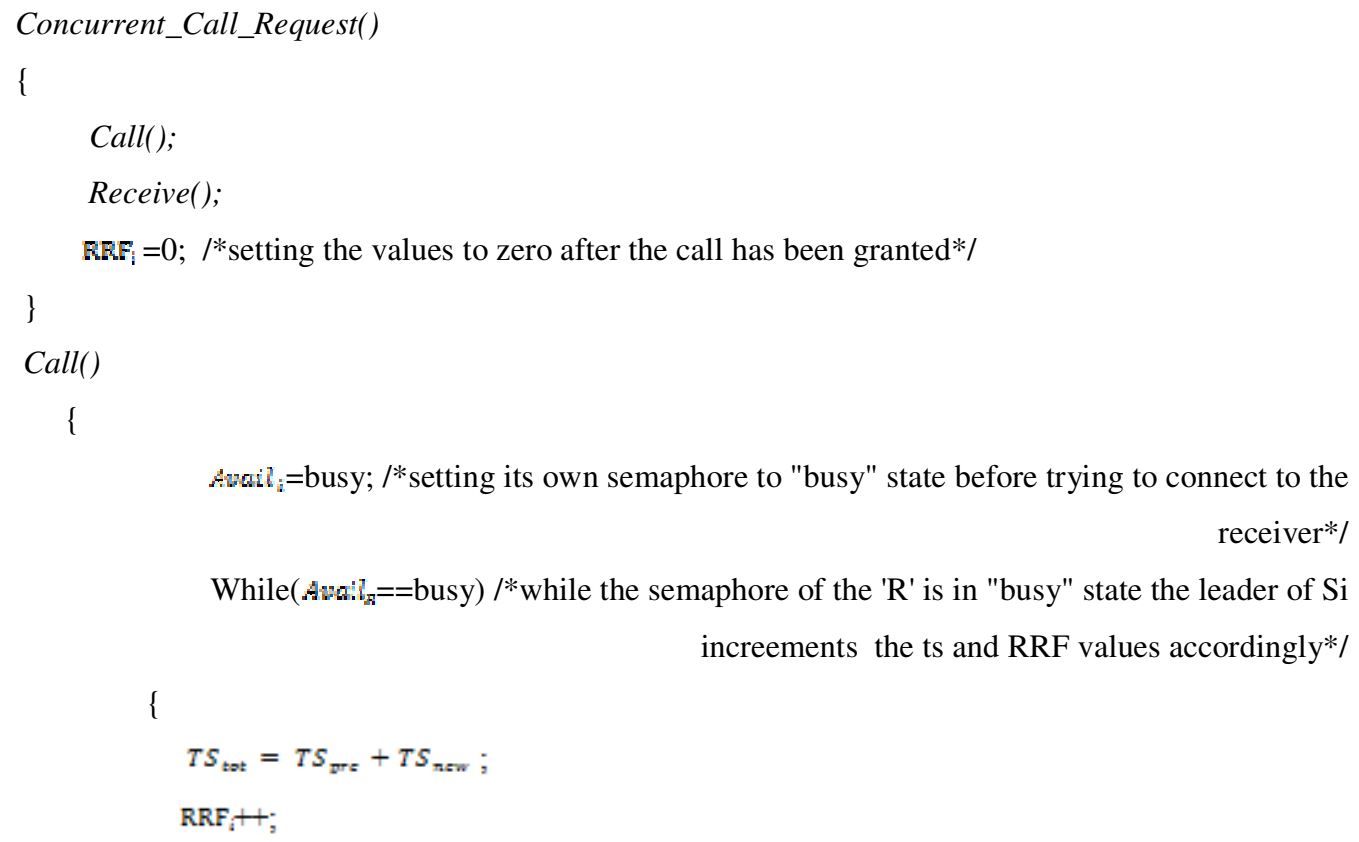

Record these values into the respective leader's data storage;

Time Complexity: The algorithm executes in $O(n)$ time for both functions call ( ) and receive ( ). 


\subsection{Example}

Initially the random available BW for call handling by $S_{1}$ and $S_{2}$ are assumed as 12 mbps and 10 mbps respectively. Similarly, the initial timestamp values for these nodes are randomly considered as 10 and 20 respectively.

STEP 1: Leaders are recording the respective values into its data storage as shown in Fig. 3.

$L S_{1}$

\begin{tabular}{|l|l|l|l|}
\hline$S_{1}$ & 5 & 10 & $\longrightarrow$
\end{tabular}

(a)

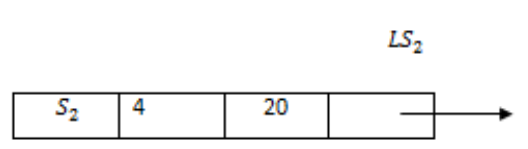

(b)

Fig. 3: After step 1: (a) Data Structure of $L S_{1}$ after entering the values; (b) Data structure of $L S_{2}$ after entering the values

STEP 2: ${ }^{L} \bar{k}$ calculates PF of each $S_{i}$ and stores them into its corresponding data storage as shown in Fig. 4.

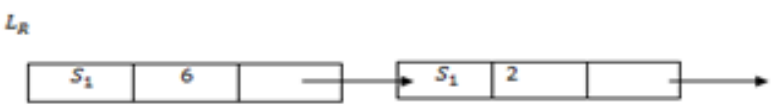

(a)

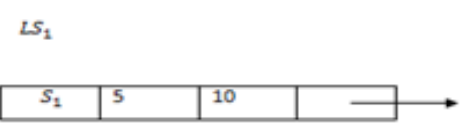

(b)

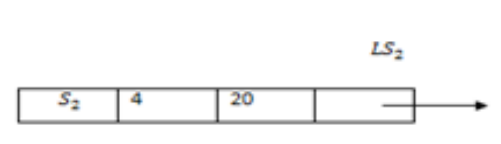

(ธ).

Fig. 4: After step 2: (a) Linked List for $L_{R}$ (b) Linked List for $L S_{1}$ (c) Linked List for $L S_{2}$

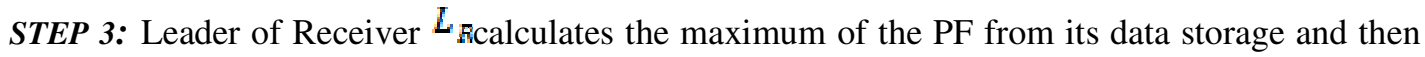
forwards the call to the respective receiver. Here, $\operatorname{PF}\left(S_{1}\right)>\operatorname{PF}\left(S_{2}\right)$. Hence $S_{1}$ 's request is granted first.

STEP 4: Set the values of $\mathrm{RRF}_{1}$ of $S_{1}$ to initial value as 0 and it is shown in Fig. 5.

$L_{R}$

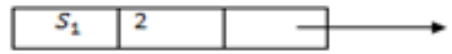

(a)

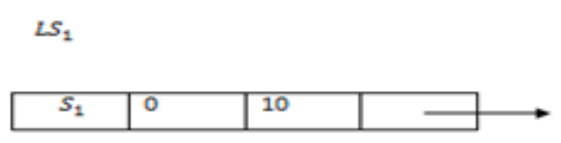

(b)

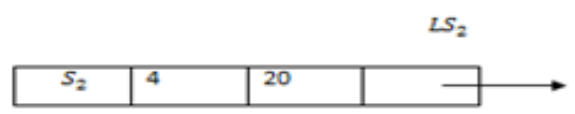

(s).

Fig. 5: After step 4: (a) Linked List for $L_{R}$ (b) Linked List for $L S_{1}$ after its call has been accepted (c) Linked List for $\mathrm{LS}_{2}$ 


\subsection{Correctness}

To prove the correctness of the proposed algorithm, a concept of progress graph [3] is used. The Progress graphs have intrinsic properties that are formalized by following postulates as given below.

P1: The concurrency state of a system defines a unique point in a progress graph.

P2: A transition from a state represented by a point $\mathrm{p} 1$ to a state represented by a point $\mathrm{p} 2$ is a ray rooted at $\mathrm{p} 1$ with direction $\mathrm{p} 1 \rightarrow$ " $\mathrm{p} 2$.

P3: A point is feasible if and only if it is not within a forbidden region. The forbidden region is the region that violates the constraints on the relative progress of the processes imposed by the signal events in progress graph.

P4: The time between two synchronizing events within each process is greater than zero.

Now, in our work, we summarize the events of the senders $\left(S_{1}\right.$ and $\left.S_{2}\right)$ as follows in table 1:

TABLE 1: Events of the senders

\begin{tabular}{|c|c|}
\hline$S_{1}$ & $S_{L}$ \\
\hline $\begin{array}{l}\mathrm{P}\left(\text { Avail }_{\mathrm{r}}\right)=\text { waiting for } \\
\text { Avail }_{\mathrm{r}} ; \\
\mathrm{V}\left(\mathrm{S}_{\mathrm{i}}\right)=\text { Signal } \mathrm{S}_{\mathrm{i}} ; \\
\mathrm{V}\left(\text { Avail }_{\mathrm{r}}\right)=\text { Signal Avail } \\
\mathrm{V}\left(\mathrm{S}_{\mathrm{i}}\right)=\text { Signal } \mathrm{S}_{\mathrm{i}} ;\end{array}$ & $\begin{array}{l}\mathrm{P}\left(\text { Avail }_{\mathrm{r}}\right)=\text { waiting for } \\
\text { Avail } \\
\mathrm{V}\left(\mathrm{S}_{\mathrm{r}}\right)=\text { Signal } \mathrm{S}_{\mathrm{i}} ; \\
\mathrm{V}\left(\text { Avail }_{\mathrm{r}}\right)=\text { Signal Avail } \\
\mathrm{V}\left(\mathrm{S}_{\mathrm{i}}\right)=\text { Signal } \mathrm{S}_{\mathrm{i}} ;\end{array}$ \\
\hline
\end{tabular}

Following these events for $S_{1}$ and $S_{2}$, the corresponding progress graph is shown in Fig. 6 . Therefore, it is clearly seen that there is neither forbidden state, nor unsafe state as there is no starvation and deadlock. Thus the concurrency among the call requests is preserved.

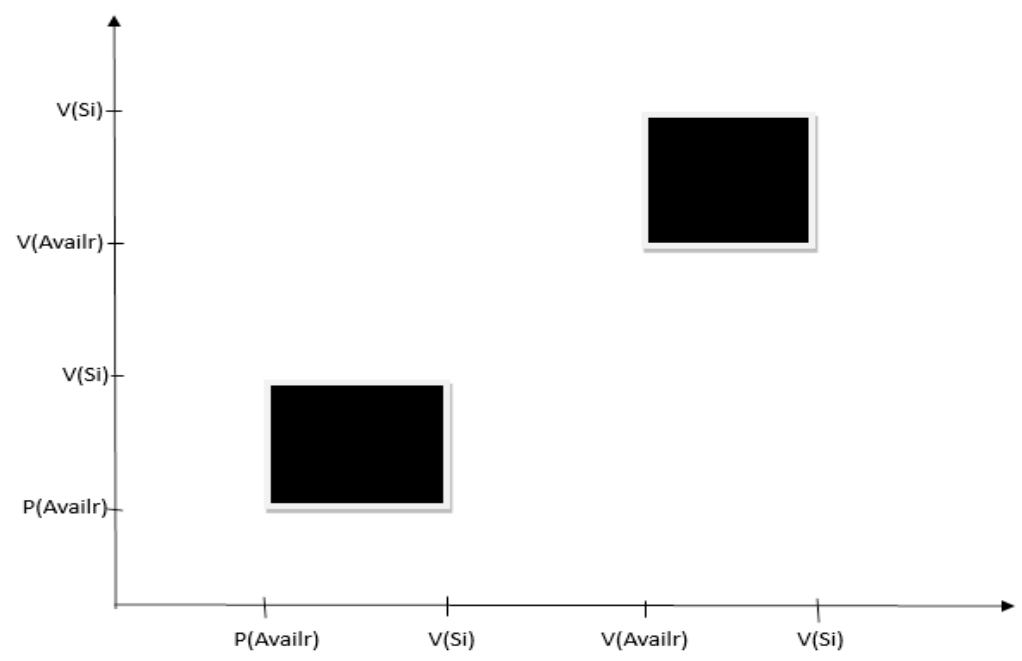

Fig. 6: Progress graphs 


\subsection{Discussion}

Presently, while handling concurrent calls no preference is given to a sender node that is repeatedly trying to connect to a particular node. So, this may lead to indefinite waiting time for such node. For illustration, considering a sender node A sending a call request to a receiver node $\mathrm{R}$ repeatedly for ( $\mathrm{n}-1)$ times and its request has not been processed yet. Now, sender node A sends the request for the nth time and concurrently a different node B sends a call request to same receiver node $\mathrm{R}$ for the first time. In such scenario, the system may usually process either of the requests without giving any preference to sender node $\mathrm{A}$.

In our proposed approach, this indefinite waiting time can be handled by assigning a RPF factor to each node of the network. This RPF factor denotes the unsuccessful attempt of a sender node. Initially, it is set to 0 and incremented by 1 after each unsuccessful attempt. So, in the above scenario, RPF factor for $\mathrm{A}$ is $\mathrm{n}$ and $\mathrm{RPF}$ factor for $\mathrm{B}$ is 1.At the receiver node $\mathrm{R}$, sender $\mathrm{A}$ is selected because of its greater RPF value

\section{CONCLUSIONS}

An efficient approach for concurrent call handling procedure is described in case of cluster based call scheduling for mobile networks. The semaphore concept is introduced here to resolve this concurrent issue. To determine the right sender among the alternatives, a priority factor (PF) is established. The several factors are determined to provide PF for a sender node. Furthermore, the proposed model executes in linear time. The performance of the model is analyzed with the help of progress graph. Moreover, we are extending our work with the help of logical clock to provide a compact and more efficient model for handling such concurrent events.

\section{REFERENCES}

[1] P.K.Guha Thakurta, Saikat Basu, Sayan Goswami and Subhansu Bandyopadhyay, "A New Approach on Cluster based Call Scheduling for Mobile Networks", Journal of Advances in Information Technology, vol. 3, no. 3, August 2012.

[2] Silberschatz, Galvin and Gagne, "Operating System Concepts”, 7th Edition, February 8, 2005.

[3] Scott D. Carson and Paul F. Reynolds, Jr., "The Geometry of Semaphore Programs", ACM Transactions on Programming Languages and Systems, Vol. 9, No. 1, January 1987, Pages 25-53.

[4] Zhengmin Kong, Liang Zhong, Guangxi Zhu, Li Yu, "A Novel Cluster-Based Routing Protocol and Cluster Reformation Criteria for Mobile Ad Hoc Networks", 2010 International Conference on Computer Application and System Modeling (ICCASM 2010).

[5] P.K.Guha Thakurta, Rajarshi Poddar and Subhansu Bandyopadhyay, "A New Approach on Coordinate based Routing Protocol for Mobile Networks", IEEE Advanced Computing Conference, February 2010. 


\section{AUTHORS}

P. K. Guha Thakurta is an Assistant is an Assistant Professor at National Institute of Technology, Durgapur for the department Computer Science and Engineering with an experience greater than eight years. He published 5 International Journal and 11International Conference papers. His area of interest include DBMS, Network, Algorithm Analysis and Design, Formal language and automata

Jahnavi Katikitala is working as a software engineer at Samsung Research India, Bangalore. She has pursued her Bachelors of Technology from National Institute of Technology, urgapur. Her area of interest include Network, Algorithm Analysis and Design \& Operating systems.

Misha Hungyo is pursuing her Masters in Computer Science and Engineering,in Motilal Nehru National Institute of Technology,Allahabad.She did her Bachelors from National Institute of Technology,Durgapur.Her area of interests are Computer Networking, Operating Systems, Data Structures and Algorithms.

Darakshan anwar is pursuing is working as a research engineer at C-DOT Bangalore.She has pursued her Bachelors of Technology degree from National Institute of Technology,Durgapur. Her area of interest include Network, Algorithm Analysis and Design.
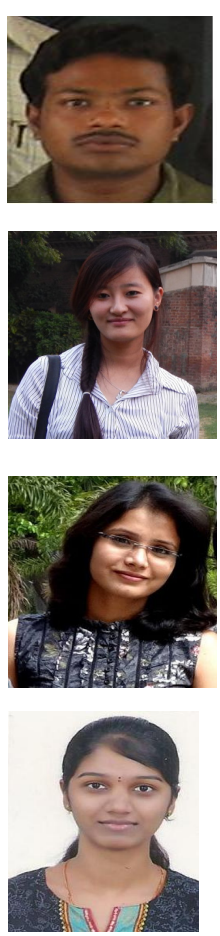